\title{
Bank Customers' Action Analysis Based on Conductive Transformation
}

\author{
Lin $\mathrm{Li}^{1{ }^{1, *}, \text { Qiongzi } \text { Qin }^{2} \text {, Xiaobo Wang }}{ }^{3}$, and Hong $\mathrm{Gao}^{4}$ \\ ${ }^{1}$ Dalian Radio and TV University, Department of Technology, Dalian, 116000, China \\ ${ }^{2}$ Hebi Senior High School, Qibin, Hebi, Henan, 458030, China \\ ${ }^{3}$ Yingdun, Yonghe, Jinjiang, Quanzhou, Fujian, 362000, China \\ ${ }^{4}$ Dalian Maritime University, Department of Mathematics, Dalian, 116026, China
}

\begin{abstract}
With the development of economic globalization, the bank market has become increasingly competitive. The bank must take measure to attract more customers, change market status and maximize the profit. Take some bank as an example, the active transformations are increasing deposit rate and decreasing loan rate. In this paper, conductive transformation is used to analyze bank customers' action changes induced by active transformations. With conductive degree, the influence of bank behavior on customer behavior can be seen. With independent degree, we can see which behavior changes can affect bank profit, and how active transformations influent bank market status.
\end{abstract}

\section{Introduction}

For the traditional banks, it is important to take some effective methods to compete with the internet finance. The important idea of Extenics is to transform the contradiction into the compatible problem by the extension transformation.

The conductive transformation is an important extension transformation[1,2]. It uses the conductive effect of the basic element to solve the contradiction problem. Because of the existence of correlation, when an object in the system is implemented with extension transformation, it can lead to the conductive transformation of the related objects in the system. We call it active transformation when it leads to the change on its action object. We call it conductive transformation when it leads to the change on its related objects [3-5].

In this paper, we take a bank as an example. We use two active transformations of increasing deposit rates and lowering interest rates, then we analyze the changes of the customers' action. By calculating the correlation degree of the customers' action we know what change of the bank customers' behavior can affect bank profit. Furthermore, the impact of the active transformation on the banking market is obtained.

\section{Conductive transformation}

Let $W=\left\{\Gamma \mid \Gamma \in\left\{B, C_{0}, k, U\right\}\right\}, \mathrm{T}$ is a set of extension transformation on $\mathrm{W}$, that is $T=\{T \mid T: W \rightarrow P(W)\}$.

For $\Gamma_{0} \in W, \varphi \in T, \varphi=\left(\Gamma_{0}, \Gamma_{0}{ }^{\prime}\right)$, if exist $\varphi_{1} \in T$,
$\Gamma \in W, \varphi_{1}=\left(\Gamma, \Gamma^{\prime}\right)$, we can get $\varphi \Rightarrow \varphi_{1} . \varphi_{1}$ is called the first order conductive transformation of $\varphi . \varphi_{1}$ is simply called conductive transformation, note $T_{\phi}$, and $\varphi$ is called active transformation.

\subsection{Conductivity}

Given the information element $I_{1}=\left(O_{1}, c_{1}, v_{1}\right)$ and $I_{2}=\left(O_{2}, c_{2}, v_{2}\right)$, if exist the conductive transformation $\varphi I_{1}=I_{1}^{\prime}=\left(O_{1}, c_{1}, v_{1}^{\prime}\right)$, for the conductive transformation ${ }_{I_{1}} T_{I_{2}}$ of $I_{2}$, we can get ${ }_{I_{1}} T_{I_{2}} I_{2}=\left(O_{2}, c_{2}, v_{2}{ }^{\prime}\right)=I_{2}^{\prime}$.

$$
v_{2}^{\prime}-v_{2}
$$

is called conductive effect;

$$
v_{1}^{\prime}-v_{1}
$$

is called active variable;

$$
\gamma=\frac{v_{2}^{\prime}-v_{2}}{\left|v_{1}^{\prime}-v_{1}\right|}
$$

is called the conductive degree of the transformation $\varphi$ on $I_{2}$.

Supposing $I_{1}, I_{2}, \ldots, I_{h}$ satisfied $L: \bigwedge_{i=1}^{h} I_{i} \sim(\ell) \hat{\wedge}_{j=1}^{k} J_{j}$, $I_{i}=\left(O_{i}, c_{i}, v_{i}\right), J_{j}=\left(j_{j}, d_{j}, u_{j}\right)$. If there are the multiple active

\footnotetext{
* Corresponding author: litiptop@ 163.com
} 
transformations, $\quad \stackrel{h}{\wedge} T_{I_{i}} I_{i}=\wedge_{i=1}^{k} I_{i}^{\prime} \quad, \quad$ occurring simultaneously. There must be a conductive transformation $\wedge_{\wedge I} T_{\wedge J_{j}}\left(\wedge_{j=1}^{k} J_{j}\right)=\wedge_{j=1}^{k} J_{j}^{\prime}$

And when $\underset{i=1}{\stackrel{h}{\wedge}} T_{I_{i}} \neq e$, the conductive knowledge on the conductive degree of each conductive information element is as follows:

$$
\gamma_{j}=\frac{u_{j}^{\prime}-u_{j}}{\sum_{i=1}^{h} \lambda_{i}\left|v_{i}^{\prime}-v_{i}\right|}
$$

where $u_{j}^{\prime}-u_{j}$ is called the conductive effect, $v_{i}^{\prime}-v_{i}$ is called the active variable, $\sum_{i=1}^{h} \lambda_{i}=1$.

\subsection{Support Degree}

In this paper, $c_{2}$ is the corresponding characteristic of $v_{2}$ and $v_{2}^{\prime}$. In (1), when $v_{2}^{\prime}-v_{2}>0, c_{2}$ is the conductive characteristics of the positive conductive effect; when $v_{2}^{\prime}-v_{2}<0, c_{2}$ is the conductive characteristics of the negative conduction effect.

For practice, we give a threshold $\delta>0$ : only when $\left|v_{2}^{\prime}-v_{2}\right|>\delta, \quad$ it is considered that $c_{2}$ the corresponding characteristic of $v_{2}$ is the effective conductive characteristic.

Characteristics $c_{2}$, that is the support of the conductive characteristics, is

$$
l_{1}=\frac{\text { Number of conduction } \text { characteristics }}{\text { Total conduction } \text { characteristic }}
$$

\section{Correlation Functions}

In the extension set, the correlation function can objectively and quantitatively describe the process of quantitative change and qualitative change[6,7]. For the evaluation characteristics of different properties, there are different forms of correlation functions, including elementary correlation function, simple correlation function, discrete correlation function, interval correlation function, etc. Select the associated function based on the requirement of the specific problem.

In this paper, elementary correlation function that the optimal point is on the right endpoint of the interval is adopted[8,9]. Given quantitative change interval and qualitative change interval matter-element respectively is $X_{0}=\langle a, b\rangle, X=\langle c, d\rangle, x_{0} \in\langle a, b\rangle, x_{0} \neq \frac{a+b}{2}$, $X_{0} \subset X$, and the public endpoint is $x_{z}$, for $x \neq x_{z}$, establish the elementary correlation function:

$$
k(x)= \begin{cases}\frac{\rho_{r}\left(x, x_{0}, X_{0}\right)}{D\left(x, X_{0}, X\right)}, & \text { others } \\ \frac{\rho_{r}\left(x, x_{0}, X_{0}\right)}{D\left(x, X_{0}, X\right)}-1, & \rho(x, X)=\rho\left(x, X_{0}\right) \text { And } x \notin X_{0}\end{cases}
$$

where

$D\left(x, X_{0}, X\right)=$

$\begin{cases}\rho(x, X)-\rho\left(x, X_{0}\right), & \rho(x, X) \neq \rho\left(x, X_{0}\right) \text { And } x \notin X_{0} \\ \rho(x, X)-\rho\left(x, X_{0}\right)+a-b, & \rho(x, X) \neq \rho\left(x, X_{0}\right) \text { And } x \in X_{0} \\ a-b, & \rho(x, X)=\rho\left(x, X_{0}\right)\end{cases}$

is the position relations of nested intervals between the point $x$ and $X_{0}, X$.

$\rho\left(x, X_{0}\right)=\left|x-\frac{a+b}{2}\right|-\frac{b-a}{2}= \begin{cases}a-x, & x \leq \frac{a+b}{2} \\ x-b, & x \geq \frac{a+b}{2}\end{cases}$

is the distance of point $x$ and interval $X_{0}$.

$\rho(x, X)=\left|x-\frac{c+d}{2}\right|-\frac{d-c}{2}= \begin{cases}c-x, & x \leq \frac{c+d}{2} \\ x-d, & x \geq \frac{c+d}{2}\end{cases}$

is the distance of point $x$ and interval $X$.

When, $x_{0}=b, \rho_{r}\left(x, b, X_{0}\right)= \begin{cases}a-x, & x<b, \\ b_{z}, & x=b, \\ x-b, & x>b,\end{cases}$

$b_{z}=\rho_{r}\left(b, b, X_{0}\right)= \begin{cases}0, & b \notin X_{0}, \\ a-b, & b \in X_{0}, \\ 0 \otimes(a-b), & b \notin X_{0} \text { And } b \in X_{0}\end{cases}$

\section{Analysis of the bank customers' action based on the conductive transformation}

In this paper, some bank as an example is taken. In order to increase the profits, the bank raises deposit rates while lowers loan rates. That is raising the current deposit rate from $0.35 \%$ to $6.35 \%$, reducing loan rate from $5.9 \%$ to $5.6 \%$.

\subsection{Determination of active transformation}

$\varphi_{1}:$ raise the current deposit rate from $0.35 \%$ to $6.35 \%$;

$\varphi_{2}$ : reduce the loan rate from $5.9 \%$ to $5.6 \%$.

The active transformation $\varphi_{1}$ and $\varphi_{2}$ involve two information elements $I_{d e p}$ and $I_{\text {loan }}$, they are expressed as a basic element

$$
\begin{array}{rlll}
I_{\text {dep }} & =\left(\begin{array}{lll}
O_{\text {dep }}, & c_{\text {dep }}, & v_{\text {dep }}
\end{array}\right) \\
& =(\text { deposit }, & \text { interestrate, } & 0.35 \%)
\end{array}
$$




$$
\begin{aligned}
& I_{\text {loan }}=\left(\begin{array}{lll}
O_{\text {loan }}, & c_{\text {loan }}, & v_{\text {loan }}
\end{array}\right) \\
& =\text { (loan, interestrate, } 5.90 \%)
\end{aligned}
$$

After active transformation:

$$
\begin{array}{rlll}
\varphi_{1} I_{\text {dep }}=I_{\text {dep }}^{\prime} & =\left(\begin{array}{lll}
O_{\text {dep }}, & c_{\text {dep }}, & v_{\text {dep }}^{\prime}
\end{array}\right) \\
& =(\text { deposit, } & \text { interestrate, } & 6.35 \%
\end{array}
$$

\subsection{Get the set of the conductive objects and the conductive feature set}

In this paper, 10 active customers, $\left\{O_{1}, O_{2}, \cdots, O_{10}\right\}$, are selected as the conductive object set in the bank's active transformation.

According to the impact on bank earnings, this paper selects five characteristics of the conductive object as the conductive feature set, that is:

(1)The Average Daily Balance $\left(c_{1}\right)$ (Ten Thousand RMB /day): The sum of the daily deposit balance of the same account divided by the amount of days in the statistics period. The average daily balance is the most important, because it directly determines how much money the banks can use, that, in turn, will affect the banks' profits.

(2) Trading Frequency(c2)(Times/Day):Total number of customer purchases over a specified period that identifies the activity level of the account.

(3) Transaction Amount(c3)Ten Thousand RMB /day): It is the total amount of money that a customer can use to purchase an enterprise product or service within a specified period of time. The transaction amount measures the fund flow of the account, which can partly reflect the liquidity of the account's owners.

(4) Personal Loan $\left(c_{4}\right)$ (Ten Thousand RMB /day): It is RMB loans issued by the bank to customers for the small and short-term or long-term large amount of funds for legitimate business activities.

(5) Customer Loyalty Degree(c5)It represents the high intensity of a customer's emotional attachment to a product or service, as well as the customer repurchasing the product. It also represents the customer recommends the product and service to others. It is easier for the bank to keep their relationship at low cost, and have new customers.

When the bank carries out the active transformation, the conductive characteristics of the customer will be transmitted. Ten customers' value of conductive characteristics before and after active transformation are in table 1.

\subsection{Result analysis}

The conductive effect of the conductive characteristics which is caused by the bank's active transformation $\varphi_{1}$ and $\varphi_{2}$ is calculated by formula (1), the results are shown in table 2 .

If $\delta=0.01$, the support degree of each conductive characteristic can be calculated by formula(5), the results are shown in table 3 .
According to formula (2), the active variables of active transformation $\varphi_{1}$ and $\varphi_{2}$ can be calculated

$\Delta v_{1}=|6.35 \%-0.35 \%|=0.06$

$\Delta v_{2}=|5.6 \%-5.9 \%|=0.003$

Adjusting deposit rate and loan rate are equally important for impacting bank earnings, so pick

$\lambda_{1}=\lambda_{2}=0.5$

According to formula of conductive degree (4), the conductivity of the $j$ th conductive characteristic is

$\gamma_{j}=\frac{u_{j}^{\prime}-u_{j}}{0.0315}, j=1,2, \cdots, 10$

The conductive degree and the conductive degree interval of the conductive object about conductive characteristic, are as shown in table 3.

As can be seen from table 2 and table 3 :

(1) "The daily balance of the account" is the conductive characteristic of positive conduction effect, the support degree is $90 \%$, which shows the change of interest rate is helpful to the daily balance deposit.

(2) "The transaction frequency" is the conductive characteristic of positive conduction effect, the support degree is $90 \%$, which shows the change of interest rate is helpful to the bank transaction frequency .

(3) "The transaction amount" is the conductive characteristic of positive conduction effect, the support degree is $90 \%$, which shows the change of interest rate is helpful for bank transaction amount.

(4) "Personal loan" is the conductive characteristic of positive conduction effect and the support degree is $100 \%$, which shows the change of interest rate has a great effect on bank personal loan.

(5) "Personal loyalty" is the conductive characteristics of positive conduction effect, the support degree is $90 \%$, which shows the change of interest rate has a great effect on bank personal loyalty.

With the active transformation of the bank, the conductivity of "the average daily balance" is between 12.70 95.23, the conductivity of "transaction frequency" is between -31.75 95.24, the conductivity of "the transaction amount" is between -3.17 44.44, the conductivity of "personal loan" is between 57.14 126.98, the conductivity of "personal loyalty" is between 31.75 126.98. All show the change of interest rate has positive conductive effect and negative conductive effect. Moreover, the support degree of the positive conductive effect about "the average daily balance", "transaction frequency", "the transaction amount", "personal loyalty" is 0.9 , the support degree of the positive conductive effect about "personal loan" is 1.0, that is, 10 customer data, 10 customer data support.

We base on the reality of the bank, for 5 conductive characteristics according to the previous data, the quantitative change interval of the average daily balance is $[0,10]$, mass interval is $[3,10]$, the optimal point is the right endpoint 10; The range of trading frequency is $[0,8]$, the qualitative change interval is $[4,8]$, the optimal point 
is the right endpoint 8; The interval of the transaction amount is $[0,3]$, the qualitative change interval is [1,3], the optimal point is the right endpoint 3; The quantitative range of personal loan is $[0,6]$ and the qualitative change interval is $[2,6]$. The quantitative range of personal loyalty is $[0,6]$, and the qualitative change interval is $[4,6]$. The optimal point is endpoint 6 .

We calculate the correlation before and after the transformation with formula (6), the result is as shown in table 4:

Knowledge from table 4 can be obtained as follows:

(1) About the average daily balance, after implementing the transformation, there are 6 data that have positive qualitative change. The correlation function value has been changed from zero to greater than 0 , and the support value is 0.6 .

(2) About the frequency of transactions, after implementing the transformation, there are 9 data that have positive qualitative change, i.e. the correlation function value has been changed from zero to greater than 0 , and the support value is 0.9 .

(3)About the transaction volume, after the transformation, 10 data has been changed, i.e. the correlation function value has been changed from zero to greater than 0 , and the support value is 1.0.
(4) About personal loans, after implementing the transformation, there are 10 data that have positive qualitative changes, namely that the value of correlation function is changed from zero to greater than 0 , and the support value is 1.0 .

(5) About the customer loyalty, after the transformation, there are 8 data that have positive qualitative changes, namely that the correlation function value has been changed from zero to greater than zero and the support value is 0.8 .

To sum up, the transformation makes three conductive characteristics "trading frequency of transactions", "Trading Volume", "personal loans" have positive qualitative change with a higher degree of support. The average daily balance support is 0.6 , showing the influence degree is different for different depositors. Such as conservative clients or degree of robustness customers affected by interest rate change is small, radical customers affected by interest rates change degree is bigger. Customer loyalty support is 0.8 , that shows the influence degree of the conductive transformation for different customers is mixed. For example, the customer 7 , customer loyalty is 6 before transformation, customer loyalty is 5 after transformation, which illustrates the high-end customer loyalty for the banks is lower than other customers.

Table 1 Values of information elements before and after transformation

\begin{tabular}{|c|c|c|c|c|c|c|c|c|c|c|}
\hline & $c_{1}$ & & $c_{2}$ & & $c_{3}$ & & $c_{4}$ & & $c_{5}$ & \\
\hline & Before & Before & Before & After & Before & After & Before & After & Before & After \\
\hline$O_{1}$ & 1.40 & 3.10 & 2.00 & 5.00 & 0.20 & 1.10 & 0.00 & 2.10 & 2.00 & 4.00 \\
\hline $\mathrm{O}_{2}$ & 2.50 & 3.50 & 3.00 & 5.00 & 0.40 & 1.50 & 0.20 & 2.20 & 3.00 & 5.00 \\
\hline $\mathrm{O}_{3}$ & 5.80 & 6.80 & 4.00 & 6.00 & 0.60 & 2.00 & 1.00 & 3.00 & 3.00 & 5.00 \\
\hline $\mathrm{O}_{4}$ & 0.50 & 2.50 & 2.00 & 5.00 & 0.05 & 1.02 & 0.00 & 2.20 & 1.00 & 5.00 \\
\hline$O_{5}$ & 2.00 & 3.80 & 3.00 & 5.00 & 0.35 & 1.60 & 0.80 & 3.00 & 3.00 & 5.00 \\
\hline$O_{6}$ & 5.00 & 8.00 & 4.00 & 7.00 & 0.90 & 1.90 & 1.00 & 5.00 & 4.00 & 6.00 \\
\hline$O_{7}$ & 9.40 & 9.00 & 6.00 & 5.00 & 1.00 & 2.50 & 2.00 & 4.00 & 6.00 & 5.00 \\
\hline$O_{8}$ & 1.80 & 3.80 & 3.00 & 5.00 & 0.30 & 1.20 & 0.00 & 2.50 & 2.00 & 5.00 \\
\hline$O_{9}$ & 2.60 & 4.50 & 2.00 & 6.00 & 0.45 & 1.45 & 1.20 & 3.00 & 3.00 & 6.00 \\
\hline$O_{10}$ & 2.70 & 4.30 & 3.00 & 5.00 & 0.55 & 1.55 & 1.40 & 3.20 & 3.00 & 5.00 \\
\hline
\end{tabular}

Table 2 The difference between the conductive characteristics of the data before and after the transformation

\begin{tabular}{|c|c|c|c|c|c|}
\hline & $c_{1}$ & $c_{2}$ & $c_{3}$ & $c_{4}$ & $c_{5}$ \\
\hline$O_{1}$ & 1.70 & 3.00 & 0.90 & 2.10 & 2.00 \\
\hline $\mathrm{O}_{2}$ & 1.00 & 2.00 & 1.10 & 2.00 & 2.00 \\
\hline $\mathrm{O}_{3}$ & 1.00 & 2.00 & 1.40 & 2.00 & 2.00 \\
\hline $\mathrm{O}_{4}$ & 2.00 & 3.00 & 0.97 & 2.20 & 4.00 \\
\hline$O_{5}$ & 1.80 & 2.00 & 1.25 & 2.20 & 2.00 \\
\hline$O_{6}$ & 3.00 & 3.00 & 1.00 & 4.00 & 2.00 \\
\hline
\end{tabular}




\begin{tabular}{llllll}
$O_{7}$ & -0.40 & -1.00 & -0.10 & 2.00 & -1.00 \\
$O_{8}$ & 2.00 & 2.00 & 0.90 & 2.50 & 3.00 \\
$O_{9}$ & 1.90 & 2.00 & 1.00 & 1.80 & 3.00 \\
$O_{10}$ & 1.60 & 2.00 & 1.00 & 1.80 & 2.00 \\
\hline
\end{tabular}

Table 3 The conductivity and conductivity interval of the conductive objects

\begin{tabular}{llllll}
\hline & $c_{1}$ & $c_{2}$ & $c_{3}$ & $c_{4}$ & $c_{5}$ \\
\hline$O_{1}$ & 53.97 & 95.24 & 28.57 & 66.67 & 63.49 \\
$O_{2}$ & 31.75 & 63.49 & 34.92 & 63.49 & 63.49 \\
$O_{3}$ & 31.75 & 63.49 & 44.44 & 63.49 & 63.49 \\
$O_{4}$ & 63.49 & 95.24 & 30.79 & 69.84 & 126.98 \\
$O_{5}$ & 57.14 & 63.49 & 39.68 & 69.84 & 63.49 \\
$O_{6}$ & 95.23 & 95.24 & 31.75 & 126.98 & 63.49 \\
$O_{7}$ & -12.70 & -31.75 & -3.17 & 63.49 & -31.75 \\
$O_{8}$ & 63.49 & 63.49 & 28.57 & 79.37 & 95.24 \\
$O_{9}$ & 60.32 & 63.49 & 31.75 & 57.14 & 95.24 \\
$O_{10}$ & 50.79 & 63.49 & 31.75 & 57.14 & 63.49 \\
{$\left[\gamma_{j \min }, \gamma_{j \max }\right]$} & {$[-12.70,95.23]$} & {$[-31.75,95.24]$} & {$[-3.17,44.44]$} & {$[57.14,126.98]$} & {$[-31.75,126.98]$} \\
$\gamma_{i j}>0$ & 0.90 & 0.90 & 0.90 & 1.00 & 0.90 \\
$\gamma_{i j}<0$ & 0.10 & 0.10 & 0.10 & 0.00 & 0.10 \\
\hline
\end{tabular}

Table 4 The correlation before and after the transformation

\begin{tabular}{|c|c|c|c|c|c|c|c|c|c|c|}
\hline & $c_{1}$ & & $c_{2}$ & & $c_{3}$ & & $c_{4}$ & & $c_{5}$ & \\
\hline & Before & After & Before & After & Before & After & Before & After & Before & After \\
\hline$O_{1}$ & $\begin{array}{l}-0.53 \\
\end{array}$ & 0.01 & -1.00 & 0.50 & -0.80 & 0.10 & -1.00 & 0.02 & $\begin{array}{l}-0.50 \\
\end{array}$ & 0.00 \\
\hline $\mathrm{O}_{2}$ & -0.17 & 0.05 & -0.33 & 0.50 & -0.60 & 0.50 & -0.90 & 0.03 & -0.25 & 0.50 \\
\hline$O_{3}$ & 0.33 & 0.54 & 0.00 & 0.50 & -0.40 & 0.50 & -0.50 & 0.17 & -0.25 & 0.50 \\
\hline $\mathrm{O}_{4}$ & -0.83 & -0.17 & -1.00 & 0.50 & -0.95 & 0.02 & -1.00 & 0.03 & -0.75 & 0.50 \\
\hline$O_{5}$ & -0.33 & 0.08 & -0.33 & 0.50 & -0.65 & 0.75 & -0.60 & 0.17 & -0.25 & 0.50 \\
\hline$O_{6}$ & 0.20 & 0.71 & 0.00 & 0.75 & -0.10 & 4.50 & -0.50 & 0.75 & 0.00 & 1.00 \\
\hline$O_{7}$ & 0.91 & 0.86 & 1.00 & 0.50 & 0.00 & 0.75 & 0.00 & 0.50 & 1.00 & 0.50 \\
\hline$O_{8}$ & -0.12 & 0.08 & -0.33 & 0.50 & -0.70 & 0.20 & -1.00 & 0.25 & -0.50 & 0.50 \\
\hline$O_{9}$ & -0.04 & 0.15 & 0.00 & 0.50 & -0.55 & 0.45 & -0.40 & 0.17 & -0.25 & 1.00 \\
\hline$O_{10}$ & -0.03 & 0.13 & -0.33 & 0.50 & -0.45 & 0.10 & -0.30 & 0.21 & -0.25 & 0.50 \\
\hline
\end{tabular}

\section{Conclusion}

In this paper, we use the theory of the conductive transformation and we analyze the conductive transformation which is caused by two active transformation of loan and deposit rate. By extracting conductive objects and conductive characteristics, calculate conductivity and support degree. Then we determine the influence degree of the active transformation of interest rate change for bank market. Through analyzing the specific case, we can get the qualitative change and quantitative change knowledge caused by conductive transformation. Furthermore, we know the influence of interest rate changes is positive for the bank, and the conductive transformation of interest rate changes is effective, which can help the bank obtain more benefits. 


\section{References}

1. Yang Chunyan, Li Xiaomei, Chen Wenwei, Cai Wen, Extension data mining methods and computerimplemented[M]. Guangdong: Guangdong Higher Education Press(2010)

2. Yang Chunyan, Cai Wen, Extension Engineering[M]. Beijing: Science Press(2007)

3. Cai Wen, Yang Chunyan, Conductive knowledge based on conductive transformation[J]. Mathematics in Practice and Theory, 38(17):85-88(2008).

4. Yang Chunyan, Li Xiaomei, Li Yang, Research on customer value based on conductive transformations $[\mathrm{J}]$. Mathematics in Practice and Theory, 42(9):142-147(2012)

5. Li Xiaomei, Yang Chunyan, Li Weihua, Conductive knowledge mining based on stock market affected by refined oil tax reform[J]. Application Research of Computers, 27(8):2865-2888(2010)

6. Cai Wen, Yang Chunyan, He Bin, Extension engineering methods $[\mathrm{M}]$. Beijing: Science Press(2003)

7. Ma Yongzhi, Ding Yi, Gao Hong, Liu Wei, Logistics market segmentation based on extension classification[C]. Information Management, Innovation Management and Industrial Engineering, 2:216-219(2013)

8. Yang Chunyan, Cai Wen, Extenics Throry, Method and Application[M]. Beijing: Science Press(2013)

9. Ding Yi, Gao Hong, Liu Wei, A personalized recommendation algorithm based on Extenics[J]. Extenics and Innovation Methods, 2013:101(2013) 